\title{
Encuesta en población abierta respecto a términos relacionados con decisiones al final de la vida
}

\author{
Luz María Guadalupe Pichardo-García, ${ }^{1}$ María de la Luz Lina Casas-Martínez, ${ }^{2}$ \\ Mónica Jaimes-Palomera, ${ }^{3}$ Alma Guadalupe Sotelo-Méndez, ${ }^{4}$ Ana Paula Sosa-Delgado, ${ }^{4}$ \\ Andrea Quintero-Luna, ${ }^{4}$ Tanit lanie López-Vergara-Anaya ${ }^{4}$ y José Alberto Aguilar-Júarez ${ }^{3}$ \\ ${ }^{1}$ Universidad Panamericana, Facultad de Derecho, Instituto Panamericano de Jurisprudencia; ${ }^{2}$ Universidad Panamericana, Facultad de Ciencias \\ de la Salud, Centro Interdisciplinario de Bioética; ${ }^{3}$ Secretaría del Medio Ambiente de la Ciudad de México, Subdirección de Análisis; ${ }^{4}$ Universidad \\ Panamericana, Facultad de Ciencias de la Salud, Escuela de Enfermería. Ciudad de México, México
}

\section{Resumen}

Introducción: Conceptos relacionados con las decisiones que se toman al final de la vida, como eutanasia, cuidados paliativos, voluntad anticipada y obstinación terapéutica son poco comprendidos por la población en general, que en el momento de enfrentar una situación terminal no está preparada para elegir la mejor opción. Objetivo: Estudio piloto $(n=544)$ para conocer lo que la población abierta entiende acerca de términos utilizados al final de la vida en cuatro ciudades de la república mexicana. Método: Encuesta vía internet de 18 preguntas sobre los distintos términos. Se trató de un estudio descriptivo, transversal, con análisis estadístico. Resultados: Se eligieron personas mayores de 18 años que no trabajaran en profesiones relacionadas con la salud. Conclusiones: La mayoría de los términos del final de la vida no interesaron ni fueron entendidos por parte de la población. El término menos reconocido fue la obstinación terapéutica (62.8 \%) y el más conocido, cuidados paliativos (91\%); se confunden los términos eutanasia y suicidio asistido (47.8\%). La edad y escolaridad resultaron de mayor influencia en los resultados, que las otras variables demográficas.

PALABRAS CLAVE: Final de la vida. Voluntad anticipada. Eutanasia. Cuidados paliativos. Obstinación terapéutica. Población abierta.

\begin{abstract}
Introduction: Concepts related to end-of-life decisions, such as euthanasia, palliative care, advance directives and therapeutic obstinacy, are poorly understood by the general population, which, when facing a terminal situation, is not prepared to choose the best option. Objective: Pilot study $(n=544)$ to find out what the open population understands about terms used in end-of-life situations in four cities of the Mexican Republic. Method: Survey via Internet with 18 questions about different terms. It was a descriptive, cross-sectional study. Statistical analysis was carried out. Results: People older than 18 years who were not engaged in health-related professional activities were selected. Conclusions: Most terms related to end-of-life decisions were found not to be interesting to or understood by a part of the population. The least recognized term was therapeutic obstinacy (62.8\%), and the most widely known, palliative care (91\%); there was confusion between the terms euthanasia and assisted suicide (47.8\%). Age and education level had more influence in the results, than other demographic variables.
\end{abstract}

KEY WORDS: End-of-life. Advance directive. Euthanasia. Palliative care. Therapeutic obstinacy. General population.

Correspondencia:

Luz María Guadalupe Pichardo-García

E-mail: Ipichard@up.edu.mx
Fecha de recepción: 13-06-2018

Fecha de aceptación: 05-01-2019

DOI:10.24875/GMM.19004465
Gac Med Mex. 2019;155:149-155

Disponible en PubMed

www.gacetamedicademexico.com 


\section{Introducción}

Para ejercer adecuadamente nuestra autonomía, uno de los aspectos más importantes es la comprensión completa de aquello que se nos cuestiona. Cuando las opciones contienen terminología específica de algún campo de conocimiento, sucede que quienes son ajenos a este comprenden poco su significado, con lo que resulta cuestionable la facultad de decisión libre e informada. Como señala Cano Valle: ${ }^{1}$

\begin{abstract}
Aunque establezcamos un enunciado conceptual sobre la libertad, en la realidad de México y, en general de América Latina, los niveles de exclusión e inequidad social van estrechamente relacionados con los diversos grados de autonomía. El bajo nivel educativo, la pobreza y la no accesibilidad a los servicios de salud constituyen una tríada perversa de aislamiento social ante la cual tenemos que luchar, ya que no puede y no debe ser un estado perpetuo el que defina a los países en desarrollo. Es urgente paliar ciertas necesidades y subsanar algunas fragilidades lo antes posible; cuanto antes, mejor que mejor.
\end{abstract}

Por este motivo, el objetivo de esta trabajo fue investigar la comprensión de los términos y posturas relacionadas con el final de la vida en la población abierta en cuatro entidades de la república mexicana.

En los últimos cinco años, en distintos países de habla hispana, en especial en México y España, se han realizado encuestas acerca del conocimiento y postura sobre términos de decisiones al final de la vida, $^{2-5}$ en las que se incluyen estudiantes universitarios, cuidadoras y médicos residentes, entre otros profesionales involucrados en la atención de la salud, pero existen muy pocas dedicadas a la población abierta, que en última instancia es la usuaria y protagonista de esas decisiones, informada por el equipo médico. Otro aspecto clave acerca de la relevancia de indagar más sobre el tema es que numerosas investigaciones se remiten a población enferma o terminal, $, 7,8$ pero no se ha explorado lo que la persona no cercana a la muerte opina sin una situación de presión.

Si bien la toma de decisiones en situaciones límite implica grandes retos ya que "la muerte, como proceso, permite prepararse para que sea acorde con las convicciones personales mantenidas a lo largo de la vida, sobre todo en aquellos casos en los que se conoce su cercanía", ${ }^{3}$ este tipo de decisiones deberían ser meditadas y comprendidas antes de una crisis, durante la cual se sobreponen numerosos factores que limitan la libertad de una persona para tomar decisiones sobre ella misma o sus allegados. ${ }^{9}$ "El tomar decisiones morales correctas ante tales circunstancias puede ser difícil, especialmente por el contenido emotivo que conllevan las situaciones límite". ${ }^{0}$ Las decisiones que se toman al final de la vida implican una importante e ineludible carga ética relaciona con el sistema de creencias de cada persona y constituyen una parte de la moral individual.

Es importante conocer la comprensión de terminología relacionada con decisiones al final de la vida en población abierta aparentemente sana, porque esta se encuentra en un estado adecuado de autonomía que le permite la reflexión. Desde la perspectiva de los investigadores, permite obtener información sin el sesgo de la angustia y estrés involucrados en decisiones extremas y evaluar el perfil de la población de las ciudades participantes para extender el número el tamaño de la muestra, incluso a más ciudades y así enfocar adecuadamente programas educativos y asistenciales ajustados a la realidad mexicana.

\section{Objetivo}

Determinar el grado de conocimientos acerca de términos involucrados en decisiones al final de la vida, que posee la población abierta en varias ciudades de México.

\section{Método}

Diseño descriptivo, exploratorio, observacional, por medio de encuestas aplicadas mediante el programa Survey Monkey, en Ciudad de México, Monterrey, Guadalajara y León, de octubre de 2017 a enero de 2018. El cuestionario consta de 18 preguntas con respuestas nominales y variables demográficas que se dividen en nominales (sexo, ciudad, religión, ocupación), ordinales (nivel de estudios y salud) y por intervalo (edad).

Los criterios de inclusión fueron:

- Mayores de 18 años.

- Individuos que desearan participación libre y voluntariamente.

- Personas que no trabajaran o hubieran trabajado en alguna profesión del sector salud.

- Individuos que no padeciera una enfermedad terminal.

Las preguntas incluyen temas acerca de la comprensión y postura de población abierta en relación con distintos términos del final de la vida. La encuesta fue anónima. El muestreo fue no probabilístico. Se realizó una encuesta piloto a 89 individuos para 
probar los reactivos. ${ }^{11} \mathrm{El}$ instrumento final se aplicó a 544 personas. Los datos colectados se analizaron por temas mediante chi cuadrada. Se realizó el análisis estadístico con el programa R-Project (versión 3.3.3, The R Foundation for Statistical Computing Platform); en la primera parte se obtuvieron frecuencias y porcentajes en la distribución de las variables demográficas y en la segunda, un análisis con tablas de contingencia para resumir la información contenida en las encuestas y medir la asociación entre las variables. Se realizó el cruce de variables, que permite determinar el grado de asociación y valorar si son estadísticamente significativas mediante chi cuadrada y su ajuste con la razón de verosimilitud, para contrastar la hipótesis de independencia entre las variables; se rechazó la hipótesis de independencia entre las variables cuando el valor de $p$ fue menor $o$ igual a 0.05 . Asimismo, se calcularon los valores del coeficiente de contingencia y Cramer para valorar el nivel de asociación, cuando se presentó dependencia entre la respuesta y las variables demográficas. Por último, se efectuó prueba de proporcionalidad en las variables sin homogeneidad.

Debido al tamaño de la muestra, el estudio se consideró piloto en las entidades federativas encuestadas.

\section{Resultados}

Contestaron la encuesta 544 personas: $70 \%$ mujeres y $30 \%$ hombres; la media de edad fue de 35 años (rango de 18 a 80 años). La distribución por grupo de edad fue la siguiente: $25 \%$ de 18 a 29 años, $10 \%$ de 30 a 39 años, $21 \%$ de 40 a 49 años, $35 \%$ de 50 a 59 años, $8 \%$ de 60 a 69 años y $1 \%$ de 70 en adelante; $83 \%$ indicó tener buena salud, $16 \%$ regular y únicamente $1 \%$, mala. Por distribución conforme a la ciudad, $58 \%$ residía en la Ciudad de México, $10 \%$ en Guadalajara, $7 \%$ en Monterrey y $25 \%$ en León. En relación con la escolaridad, la licenciatura constituyó $50 \%$, el posgrado $23 \%$, preparatoria o equivalente $14 \%$, carrera técnica $10 \%$, primaria y secundaria $3 \%$. En cuanto a la ocupación, $26 \%$ practicaba una profesión, $16 \%$ ama de casa, $16 \%$ estudiante, $13 \%$ empleado, $7 \%$ se dedicaba a otra actividad, $5 \%$ servidor público, $5 \%$ administrativo, $4 \%$ pensionado, $4 \%$ comerciante, $2 \%$ secretaria y $1 \%$ empleada del hogar.

Las respuestas por tema permitieron percibir en qué áreas se tiene que trabajar para aclarar los términos. Las frecuencias se muestran en la Tabla 1.
En el Anexo 1 se indican las variables demográficas significativas que influyeron por tema $(p<0.05)$, se presenta el nivel de significancia obtenido mediante el análisis de las tablas de contingencia para medir el grado de asociación de cada pregunta y las variables demográficas y se indican las variables con relación positiva, que constituye parte de la valoración de resultados de este trabajo.

Como resumen señalamos los siguientes datos relevantes:

1. Las personas entre 40 y 69 años, así como los profesionales conocieron bien el término cuidados paliativos, el cual resultó indiferente para los estudiantes y menores de 29 años.

2. Existió confusión en la población respecto a los términos eutanasia y suicidio asistido. Para eutanasia, las variables más significativas fueron la edad y la religión. Los encuestados con posgrado diferenciaron adecuadamente el término.

3. Sobre el término obstinación terapéutica, a mayor grado de escolaridad, mayor desacuerdo con su aplicación. Las personas mayores de 50 años no lo comprendieron.

4. Los encuestados con menor grado de educación, entre 18 y 39 años y estudiantes, no conocieron el término voluntad anticipada o fueron indiferentes. El resto de la población lo entendía y aceptaba (88\%).

5. Respecto a la relevancia del conocimiento de términos relacionados con el final de la vida, $90 \%$ coincidió en que facilita las decisiones en situaciones críticas. Las personas menores de 30 años, así como los estudiantes manifestaron indiferencia. Las variables más asociadas fueron edad, ocupación, nivel de escolaridad y religión; $77 \%$ de los encuestados señaló que no recibía información útil sobre estos términos por parte del personal de salud o la recibía parcialmente.

\section{Discusión}

Este estudio exploró los conocimientos de la población en general en la Ciudad de México, León, Guadalajara y Monterrey, en encuestados que se consideraban sanos (83\%), quienes desconocieron y confundieron la mayoría de los términos relacionados con el final de la vida, por lo que es urgente cubrir ese vacío conceptual a fin de promover decisiones informadas en la población general.

Respecto a comprensión de términos, el menos conocido fue obstinación terapéutica (63\%) y el más 
Tabla 1. Resultados de la encuesta piloto sobre el conocimiento de términos relacionados con el final de la vida $(n=544)$

\begin{tabular}{|c|c|c|c|}
\hline & $\begin{array}{c}\text { De acuerdo } \\
(\%)\end{array}$ & $\begin{array}{c}\text { Indiferente } \\
(\%)\end{array}$ & $\begin{array}{c}\text { En desacuerdo } \\
(\%)\end{array}$ \\
\hline \multicolumn{4}{|l|}{ Tema 1. Cuidados paliativos } \\
\hline P12. ¿Sabes qué son los cuidados paliativos? & 71 & - & 29 \\
\hline $\begin{array}{l}\text { P14. "Los cuidados paliativos consisten en los apoyos adecuados, médicos, } \\
\text { psicológicos, familiares, espirituales en enfermos terminales, para que la muerte } \\
\text { sea un trance menos doloroso y el duelo sea más tranquilo y en paz". }\end{array}$ & 92 & 6 & 2 \\
\hline $\begin{array}{l}\text { P19. El apoyo de los cuidados paliativos en enfermos terminales puede ayudar en } \\
\text { muchos casos a encontrar sentido a esa última etapa de la vida y vivirla sin } \\
\text { angustia y con más serenidad }\end{array}$ & 92 & 5 & 3 \\
\hline
\end{tabular}

Tema 2. Eutanasia y suicidio asistido

P9. ¿Sabes lo que es el suicidio asistido?

$\begin{array}{lll}76 & - & 24 \\ 96 & - & 4 \\ 48 & - & 52 \\ 74 & 7 & 19\end{array}$

P10. ¿Sabes qué es la eutanasia?

P11. ¿Sabes si son lo mismo la eutanasia y el suicidio asistido?

P13. ¿Cuál es tu opinión sobre la siguiente definición de eutanasia o suicidio asistido: "Toda acción u omisión a petición del paciente que provoque deliberadamente su muerte con el fin que no presente dolor o sufrimiento"?

P20. Un paciente en fase terminal puede no estar en condiciones mentales suficientemente lúcidas para pedir la eutanasia.

P21. Considero que la vida de una persona debe ser respetada hasta su fin natural. Nunca sería admisible que el médico aplique eutanasia, aunque el paciente lo pida.

P22. Según mi criterio es preferible morir a vivir sin una calidad de vida adecuada.

$60 \quad 9 \quad 31$

\section{Tema 3. Obstinación terapéutica}

P15. ¿Sabes qué es la obstinación terapéutica?

$38-62$

P16. La obstinación terapéutica consiste en continuar utilizando tratamientos que no sirven ya al paciente terminal en ningún sentido.

53

48

70 aunque no tenga posibilidades de recuperación.

P18. No es ético continuar aplicando obstinadamente medidas que ya no pueden ser útiles en pacientes terminales.

\section{Tema 4. Voluntad anticipada}

P23. La Voluntad Anticipada es un documento legal que sirve para la toma de decisiones sobre los tratamientos que se desea se apliquen y cuáles no, en caso de perder la conciencia y estar en fase terminal.

P24. El uso del documento de Voluntad Anticipada ayuda a evitar problemas en las decisiones que se toman en enfermos terminales.

\section{Tema 5. Conocimiento general de términos}

P25. ¿Si conoces estos términos te facilitará tomar decisiones serenas y oportunas con el objeto de evitar y controlar los síntomas de esas etapas, como el dolor, el sufrimiento, la ansiedad y la depresión, tanto si tú fueras el enfermo o uno de tus familiares cercanos?

\begin{tabular}{lccc}
\hline & Sí (\%) & No (\%) & A veces (\%) \\
\hline $\begin{array}{l}\text { P26. El personal de salud habitualmente informa de manera completa y clara acerca } \\
\text { de lo que necesitas saber para tomar la mejor decisión para ti o para tu paciente } \\
\text { en la última fase de la vida. }\end{array}$ & 22 & 28 & 50 \\
\hline
\end{tabular}

$87 \quad 6$

$\begin{array}{lll}88 & 5 & 7\end{array}$

5

6 
conocido, cuidados paliativos (91\%). El término eutanasia es suficientemente conocido (75\%).

Se exploró la indiferencia hacia ciertos temas: encontramos más indiferencia hacia obstinación terapéutica $(20 \%)$, lo cual concuerda con la falta de comprensión. Los resultados concuerdan con la opinión de Font Busquets et al., quienes señalan que "es necesario encontrar estrategias que disminuyan esta disociación, entre ellas mayor implicación de los profesionales asistenciales en la ayuda a la planificación anticipada de la atención y la elaboración del documento de voluntades anticipadas". ${ }^{12}$

La obstinación terapéutica, definida en términos éticos, fue rechazada por la mayoría. Estos resultados coinciden con los de Ortiz Gonçalves et al.,13 quienes señalan que más de $50 \%$ de la población está en contra de la obstinación terapéutica. En otros temas, la indiferencia osciló entre 5 y $10 \%$ (Tabla 1), significativa desde la perspectiva de la comprensión de términos, pues debería ser casi nula. Se puede inducir que los temas estuvieron lejos de los intereses de este tipo de población en las circunstancias que estaban viviendo al momento de la encuesta.

La tendencia a favor de la eutanasia fue de $50 \%$ y $40 \%$ en contra. Si bien mucho se ha escrito sobre el tema, como señalan Morales et al., "en cambio, apenas hay estudios sobre cuidados paliativos, suicidio médicamente asistido y ayuda espiritual". ${ }^{14}$ Esta tendencia no es exclusiva de la población general, como se aprecia en el estudio de Toro Flores et al., ${ }^{15}$ quienes señalan que $47 \%$ de los médicos pediatras considera la eutanasia como una práctica aceptable.

Es importante destacar que cuando se abordó el tema de cuidados paliativos oportunamente ofrecidos, la mayoría poblacional estuvo de acuerdo con esta medida (91\%). Por el tipo de respuestas sobre eutanasia y cuidados paliativos, puede inferirse que la población estaba dividida respecto a la eutanasia, pero a favor mayoritario de los cuidados paliativos si se ofrecen oportunamente. Este es un punto muy importante por considerar en las políticas públicas en salud, especialmente ahora que existe la obligatoriedad de contar con el servicio de cuidados paliativos en México. La accesibilidad y calidad del servicio seguramente serán decisivas para que la población se incline a una u otra decisión al final de la vida, en la cual mucho tiene que ver la información que otorga el personal de salud.

Una propuesta muy acertada es la de Vermogeen et al.:16

\begin{abstract}
Con el fin de mejorar la calidad de la atención al final de la vida de los miembros de la familia, las habilidades de comunicación de los profesionales de atención individual necesitan ser mejoradas a través de cambios en la educación médica y programas de capacitación (...) la importancia de promover una cultura de la asistencia sanitaria donde los profesionales de la atención tengan en cuenta los intereses de la persona que padece una enfermedad y de sus familiares, práctica habitual en los cuidados paliativos. Se necesita investigación a futuro para identificar intervenciones que puedan promover una perspectiva holística dentro y entre los diferentes servicios de atención médica.
\end{abstract}

Se puede concluir que las variables sexo y nivel educativo tienen influencia importante en preguntas enfocadas a conceptos o definiciones; a diferencia de las preguntas en relación con la vida y la muerte, en las cuales tienen influencia particular la ciudad y la religión.

Estos temas no explorados anteriormente en encuestas mexicanas tienen relevancia funcional. A mayor escolaridad, mayor comprensión de la terminología, lo cual indica que hay que desarrollar estrategias para la educación sobre estos términos en la población de baja escolaridad. Al revisar la bibliografía sobre el tema en población abierta, solamente se encontró un artículo en estudiantes, ${ }^{9}$ en 2007 , orientado a la voluntad anticipada y que aborda otros términos relacionados con decisiones el final de la vida; también fue clara la falta de conocimiento.

La encuesta muestra que solo $30 \%$ de los encuestados consideró que el personal de salud proporciona información adecuada para elegir los tratamientos más acertados en enfermos terminales. Este punto, eminentemente ético, implica una deficiente relación médico-paciente e incumple especialmente los principios bioéticos de beneficencia y no maleficencia.

Una de las limitaciones del estudio fue la desproporción de la muestra en algunos estados, que se compensó con la homogenización para las comparaciones entre variables demográficas. La mayor fortaleza estuvo constituida por la estratificación de los resultados conforme a las variables demográficas, principalmente edad, religión, ocupación y nivel de escolaridad, pues estos parámetros no habían sido estudiados adecuadamente por otros investigadores en México.

\section{Conclusiones}

La preparación para tomar una decisión autónoma y ponderada inicia mucho antes de que se presente una situación crítica. Por ello, es urgente diseñar e impulsar 
campañas educativas para promover una cultura del bien morir en personas sanas, quienes pueden reflexionar con antelación sobre términos al final de la vida, adaptados a edad, ocupación, escolaridad, sexo, religión, valores, cultura, en diferentes medios. Deberá investigarse en posteriores encuestas cómo, dónde, cuándo y quién se encargaría de esta tarea, que permitiría un tránsito más natural y sin angustias en el momento inevitable de la muerte, parte esencial del vivir.

\section{Financiamiento}

Esta investigación ha sido financiada por la Universidad Panamericana a través del Fomento a la Investigación UP 2017, código UP-Cl-2017-DER.

\section{Bibliografía}

1. Cano-Valle F. ¿Es posible avanzar hacia una muerte digna?: la situación en México comparada con la situación en Holanda. Rev Inst Nal En Resp Mex. 2007;20:5-8.

2. Cucalón-Arenal JM, Marín-Ibáñez A, Cía-Gómez P, Blay-Cortés MG Opinión de los universitarios sobre los cuidados al final de la vida. FEM 2013;16:181-186.

3. Ibáñez-Masero O, Ortega-Galán AM, Carmona-Rega MI, Ruiz-Fernández MD, Cabrera-Troya J, García-Cisneros R, et al. El significado de morir dignamente desde la perspectiva de las personas cuidadoras: estudio fenomenológico. Enferm Clin. 2016;26:333-402.

4. Gaona-Flores VA, Campos-Navarro LA, Ocampo-Martínez J, Alcalá-Martínez E, Patiño-Pozas M. La "voluntad anticipada" y su conocimiento por médicos en hospitales de tercer nivel. Gac Med Mex. 2016;152:486-494.
5. Hildén HM, Louhiala P, Palo J. End of life decisions: attitudes of Finnish physicians. J Med Ethics. 2004;30:362-365.

6. Sarmiento-Medina M, Vargas-Cruz S, Velásquez-Jiménez C, Sierra de Jaramillo M, Problemas y decisiones al final de la vida en pacientes con enfermedad en etapa terminal. Salud Publica. 2012:14:116-128.

7. Garduño-Espinosa A, Ham-Mancilla O, Méndez-Venegas J, Niembro ZAM, Pando CJM, Martínez BR, et al. Decisiones médicas al final de la vida. Recomendaciones para la atención a pacientes con enfermedades en estado terminal. Acta Pediatr Mex. 2006;27:307-316.

8. Tejedor-Torres JC, López de Heredia-Goya J, Herranz-Rubia N, Nicolás-Jiménez $\mathrm{P}$, García-Muñoz $\mathrm{F}$, Pérez-Rodríguez J, et al. Recomendaciones sobre toma de decisiones y cuidados al final de la vida en neonatología. An Pediatr (Barc). 2013;78:190.e1-190.e14.

9. Casas ML. Encuesta en población abierta sobre contenidos aplicables a los documentos de voluntades anticipadas (DVA). Rev Conamed. 2007;12:9-15

10. Casas ML. Detección de necesidades familiares en la atención a pacientes en estado terminal y crónico en la Ciudad de México. Rev Conamed. 2006;11:19-24.

11. Pichardo-García LM, Casas-Martínez ML, Jaimes-Palomera M, Sotelo-Méndez AG, Sosa-Delgado AP, Quintero- Luna A, et al. Términos que facilitan las decisiones de fin de vida. ¿Es necesario prepararse para una buena muerte? An Med Hosp ABC. 2017;62:266-270.

12. Font-Busquets JM, De la Fuente Hidoine M, Lushchenkova O, Quintana S. Conocimiento y valoración por parte de los ciudadanos de Cataluña sobre las voluntades anticipadas y la atención al final de la vida. Medi Pa. 2014:21:153-159.

13. Ortiz-Gonçalves B, Santiago-Sáez A, Albarrán-Juan E, Labajo-González E, Perea-Pérez B. Elaboración de un cuestionario sobre conocimientos y actitudes de la población madrileña frente al final de la vida. Gaceta San. 2018;32:319-402.

14. Morales-Valdés G, Alvarado-Romero T, Zuleta-Castro R. Limitación del esfuerzo terapéutico en cuidados intensivos pediátricos: conocimiento y actitudes bioéticas del profesional médico. Rev Chil Pediatr. 2016;87: 116-200.

15. Toro-Flores R, López-González R, López-Muñoz JA. Conocimientos y actitudes de los pacientes críticos y sus familiares respecto a las directivas anticipadas y la toma de decisiones al final de la vida. Enferm Intensiva. 2017;28:21-30.

16. Vermorgeen $M$, De Vleminck A, Deliens L, Houttekier D, Spruytte N, Van-Audenhove $\mathrm{C}$, et al. Do physicians discuss end-of-life decisions with family members? A mortality follows back study. Patient Educ Couns. 2018;101:1378-1384. 
Anexo 1. Valores de significancia entre las variables demográficas y cada una de las preguntas*

Pregunta

P09. ¿Sabes lo que es el suicidio asistido?

P10. ¿Sabes lo que es la eutanasia?

P11. ¿Sabes si son lo mismo la eutanasia y el suicidio asistido?

P12. ¿Sabes lo que son los cuidados paliativos?

P13. ¿Cuál es tu opinión sobre la siguiente definición de eutanasia o suicidio asistido: "toda acción u omisión a petición del paciente que provoque deliberadamente su muerte con el fin de que no presente dolor o sufrimiento"?

P14. "Los cuidados paliativos consisten en los apoyos adecuados, médicos, psicológicos, familiares, espirituales en enfermos terminales, para que la muerte sea un trance menos doloroso y el duelo sea más tranquilo y en paz".

P15. ¿Sabes lo qué es la obstinación terapéutica?

P16. La obstinación terapéutica consiste en continuar utilizando tratamientos que no sirven ya al paciente terminal en ningún sentido.

P17. El equipo médico debe luchar por la vida del paciente hasta el último instante, aunque no tenga posibilidades de recuperación.

P18. No es ético continuar aplicando obstinadamente medidas que ya no pueden ser útiles en pacientes terminales.

P19. El apoyo de los cuidados paliativos en enfermos terminales puede ayudar en muchos casos, a encontrar sentido a esa última etapa de la vida y vivirla sin angustia y con más serenidad.

P20. Un paciente en fase terminal puede no estar en condiciones mentales suficientemente lúcidas para pedir la eutanasia.

P21. Considero que la vida de una persona debe ser respetada hasta su fin natural. Nunca sería admisible que el médico aplique la eutanasia, aunque el paciente lo pida.

P22. Según mi criterio es preferible morir a vivir sin una calidad de vida adecuada.

P23. La Voluntad Anticipada es un documento legal que sirve para la toma de decisiones sobre los tratamientos que se desea se apliquen y cuáles no, en caso de perder la conciencia y estar en fase terminal.

P24. El uso del documento de Voluntad Anticipada ayuda a evitar problemas en las decisiones que se toman en enfermos terminales.

P25. ¿Si conoces estos términos te facilitaría tomar decisiones serenas y oportunas con el objeto de evitar y controlar los síntomas de esa etapa, como son el dolor, el sufrimiento, la ansiedad y la depresión, tanto si tú fueras el enfermo o uno de tus familiares cercanos?

P26. El personal de salud habitualmente te informa de manera completa y clara acerca de lo que necesitas saber para tomar la mejor decisión para ti o para tu paciente en la última fase de la vida.

Ciudad Escolaridad Salud Sexo Religión Edad Ocupación Total

$\begin{array}{ccccc} & & & 0 \\ 1 & & 1 & & 1 \\ 1 & & & & 1 \\ 1 & 1 & & 1 & 4 \\ 1 & & & 1 & 3\end{array}$

